\section{Electronic bed-wetting alarm and toilet trainer}

In many children with enuresis repetitive awakening by an alarm as soon as urination begins will gradually train the brain to exert automatic control over the bladder. The child will eventually either wake up before urination or sleep throughout the night without needing to empty the bladder. Such treatment is accepted as the most successful way of curing bed-wetting and may result in over $80 \%$ success within a few months. ${ }^{1} \mathrm{~A}$ few children become adolescent and then adult without any of the methods available up until now achieving dry nights.

\section{New device and method of use}

Existing devices are cumbersome and expensive. They need careful tuition and intensive follow-up to achieve a good rate of success. These are not always available and failure is common. An alternative device has been developed which has many advantages over the traditional bell and

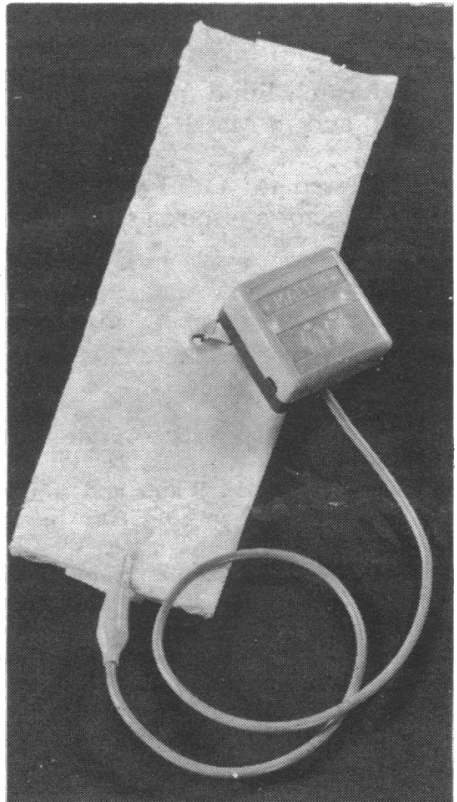

Electronic bed-wetting alarm. pad. It is a matchbox-size, lightweight $(50 \mathrm{~g})$ alarm that may be worn unobtrusively on the patient. The alarm is permanently connected to a small, injection-moulded, moisture-sensitive plate placed inside an absorbent, disposable press-on towel with no contact between sensor and skin. With the onset of urination this sensor comes in contact with moisture and a loud buzzer is activated.

The alarm uses hygienic, cheap, and readily available press-on towels and is permanently connected to the sensor so that accidental separation cannot occur. The device is triggered as soon as the absorbent press-on towel is moistened, so that actual wetting of the bed may be avoided. The alarm automatically resets and can be used repeatedly during the same night without parental help. The alarm has no on/off switch and the patient cannot forget to switch it on or switch it off and go back to sleep as only drying the sensor inactivates the alarm. The alarm is easily portable and may be used away from home. It may be worn during the day for toilet training of the mentally handicapped and may also have a role in geriatrics to alert nurses to the time of micturition.

Case history-A 12-year-old girl presented in 1974 with enuresis, having failed to respond to any of the usual remedies. Over the next eight years she was seen by many doctors and specialists. Proteinuria of minimal-change glomerulonephritis was treated, but enuresis continued whether laboratory measurements reflecting her nephrotic syndrome were normal or abnormal and despite attempts to cure or modify with tricyclics, diuretics, ephedrine, and a trial with the bell and pad. In November 1981 she was issued with the alarm described with written instructions only. She was woken by the alarm, presumably as a result of some loss of urine but not enough to wet the bed. She then micturated normally and returned to her dry bed. After some weeks she found herself waking before the alarm and was hoping soon to be confident enough to return the alarm. She was delighted with this, awakening for the first time in her life in nightclothes and a bed not smelling and saturated with urine. She had slept away from home for the first time, though she was still choosing friends who were aware of her problem, and two months later was considering a holiday. We are aware of many successes with patients using this alarm, but know of no more impressive example of success where other treatments have failed.

The equipment has now been issued to more than 100 Nottingham children with enuresis, some attending hospital, some local authority clinics, and some acquiring the equipment without intervention of medical personnel. Many successes, no failures, and no complaints have been reported. Those with experience of other equipment had a strong preference for the new device. Details of the first 15 patients to be treated-their age, sex, and the duration in weeks of treatment until they were dry-are as follows: age $11(\mathrm{~F})$ 18 weeks; age 11 (M) 26 weeks; age 12 (M) 26 weeks; age 10 (M) 31 weeks; age $9(\mathrm{M})$ eight weeks; age 10 (F) 12 weeks; age 8 (F) two weeks; age 14 (F) 15 weeks; age $14(\mathrm{~F}) 19$ weeks; age $7(\mathrm{~F}) 13$ weeks; age $14(\mathrm{~F})$ two weeks; age $16(\mathrm{M})$ three weeks; age $16(\mathrm{M})$ five weeks; age $8(\mathrm{M})$ four weeks; age 20 (F) eight weeks.

\section{Comment}

The device described is safe and effective. Those with experience of alternative systems have no doubt about the merits of this new device, which is much simpler to use and is neither cumbersome nor intrusive. The patient wears normal nightclothes, in contrast to using the bell-and-pad method when it is recommended that no "bottoms" should be worn. It is cheaper to buy, easier to store, and no staff time is needed for instruction or supervision. Direct sales to the public, without medical supervision and without incident, have now been taking place for nine months, after initial successful trials under medical supervision. There may be objections to this approach, but much medical and nursing time is saved by reducing the need for consultation and instruction. Presentation of renal or other disease as enuresis is rare, and will still be detectable when failure of this device to train a child results in medical consultation. Improved results in treating enuresis should reduce stress for children and parents̀.

${ }^{1}$ Meadow R. How to use buzzer alarms to cure bed-wetting. $\mathrm{Br}$ Med $\mathfrak{f}$ 1977; ii:1073-5.

(Accepted 5 April 1982)

Renal and Paediatric Units, City Hospital, Nottingham NG5 1PD H MALEM, PHD, research fellow

M S KNAPP, MD, FRCP, consultant physician

E J HILLER, MB, FRCP, consultant paediatrician

\section{$\theta$ \\ Cryptosporidial enteritis without general or selective immune deficiency}

Recent reports have described an acute enterocolitis associated with infection by the protozoan cryptosporidium. ${ }^{1-4}$ Infection by a wide range of organisms often develops in immunologically deficient patients. We describe a case of intestinal cryptosporidiosis with only mild variations from normal immunological variables.

\section{Case report}

A 24-year-old woman was admitted in June 1979 with a five-day history of colicky central abdominal pain and nausea and having passed frequen greenish watery stools. She had not been abroad and had no history of atopy but had been admitted 10 years earlier for abdominal pain, which had settled after 24 hours. On examination her temperature was $38.2^{\circ} \mathrm{C}$ and she had mild generalised abdominal tenderness, with some guarding and rebound tenderness in the right iliac fossa. Rectal examination showed generalised tenderness, worse on the right. White cell count was $6.0 \times 10^{9} / 1$ and haemoglobin concentration $13.4 \mathrm{~g} / \mathrm{dl}$. A limited right hemicolectomy was performed at laparotomy, the ileum appearing thickened and congested, clinically suggesting acute Crohn's disease. She recovered with no further abdominal pain or diarrhoea and two years later remained well. A recta biopsy specimen was taken at routine follow-up a month after discharge.

Macroscopically the terminal ileum and caecum had mildly congested serosa while the bowel wall and mucosa appeared normal. Histologically the mucosa of both ileum and caecum showed mild inflammatory changes with crypt abscesses but no ulceration, granuloma formation, or extension of inflammation into the submucosa. Peyer's patches in the ileum were prominent with germinal centres, and there was reactive follicular hyperplasia of the draining lymph nodes. The surface epithelium of the ileal and caecal mucosa was covered with small haematoxyphil bodies, which on transmission electron microscopy were protozoa 3-4 $\mu \mathrm{m}$ in diameter attached to the microvillar surface of mucosal cells (figure). These were identified as cryptosporidia.

The rectal biopsy specimen taken at follow-up showed a normal, un- 